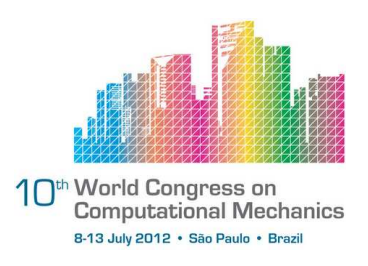

\title{
COMPUTATIONAL PREDICTION OF WIND INDUCED VIRATIONS IN SILO GROUPS USING 2D AND 3D CFD SIMULATIONS
}

\author{
J. Hillewaere ${ }^{1}$, J. Degroote ${ }^{2}$, G. Lombaert ${ }^{1}$, J. Vierendeels ${ }^{2}$, G. Degrande ${ }^{1}$ \\ ${ }^{1}$ KU Leuven, Department of Civil Engineering, Kasteelpark Arenberg 40, B-3001 Heverlee, \\ Belgium (jeroen.hillewaere@bwk.kuleuven.be) \\ ${ }^{2}$ Ghent University, Department of Flow, Heat and Combustion Mechanics, St. Pietersnieuw- \\ straat 41, B-9000 Gent, Belgium
}

\begin{abstract}
Wind induced ovalling vibrations were observed during a storm in October 2002 on several empty silos of a closely spaced group consisting of 8 by 5 thin-walled silos in the port of Antwerp (Belgium). To clarify the cause and location of the observed silo vibrations, a thorough analysis of the aerodynamic pressures on the silo surfaces is required. Therefore, both $2 D$ and $3 D$ computational fluid dynamics (CFD) simulations have been performed. While the 2D simulations mainly aim at studying the influence of the angle of incidence of the wind flow on the location where ovalling vibrations can be observed, the $3 D$ simulations are performed to incorporate $3 D$ flow effects into the analysis and to assess the validity of the conclusions of the $2 D$ simulations. The $3 D$ pressure distribution on the silo walls is applied as an external time dependent load on a 3D finite element model of a silo to determine the structural response. Afterwards, modal projection of the load is performed to determine the contribution of each ovalling mode shape to the dynamic structural response. For the $2 D$ simulations, a similar technique of harmonic decomposition is derived and validated with the $3 D$ one-way coupling approach. The results of both approaches yield evidence of the onset of ovalling vibrations, corresponding to the observed pattern of oscillations in the Antwerp silo group.
\end{abstract}

Keywords: Ovalling, silo group, aerodynamic pressures, wind induced vibration.

\section{INTRODUCTION}

Wind induced ovalling vibrations were observed during a storm in October 2002 on several empty silos of a closely spaced group consisting of 8 by 5 thin-walled silos in the port of Antwerp (Belgium, figure 1). The ovalling vibrations were observed near the lee side corner of the silo group (i.e. silo 40 and neighbouring silos $24,32,38$, 39, etc., figure 2). To clarify the underlying mechanisms inducing this aeroelastic phenomenon, a thorough understanding of the fluid flow around such groups and of the dynamic pressure loads on the silo walls is 
first required. To this end, 2D and 3D computational fluid dynamics (CFD) simulations are performed.

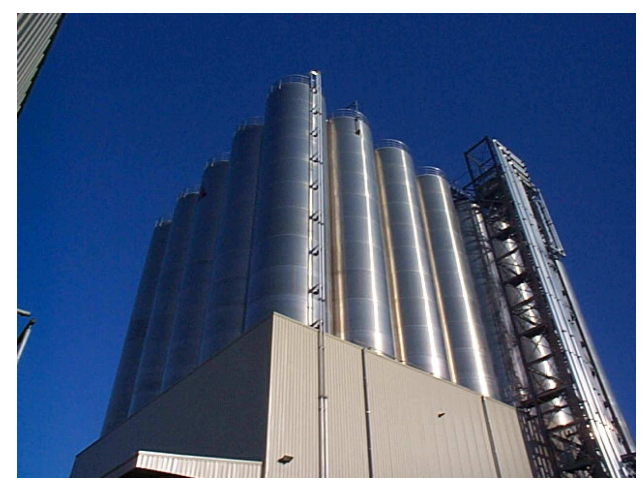

Figure 1. Photo of the 8 by 5 silo group in Antwerp.

The dynamic properties of the silo structures will be presented first. Finite element (FE) modeling is used to determine natural frequencies and ovalling mode shapes of a silo. The numerical modeling of the highly turbulent flow around the silo group in both $2 \mathrm{D}$ and 3D simulations is presented in the third section. In addition to the description of the applied numerical procedures and the approach to verify and validate the simulation results, a subsection is dedicated to the modeling of turbulence at the inlet boundary in both $2 \mathrm{D}$ and $3 \mathrm{D}$ simulations and its importance for the simulation results. In the last section of this paper, the structural response to the calculated aerodynamic pressure loads is investigated. The 3D transient wind pressure distribution is applied as an external time dependent load on the 3D FE model of a silo. Modal decomposition of the 3D pressure loads is used to examine the contribution of each ovalling mode shape in the dynamic structural response, while a related technique of harmonic decomposition for the 2D pressure loads is also derived and validated.

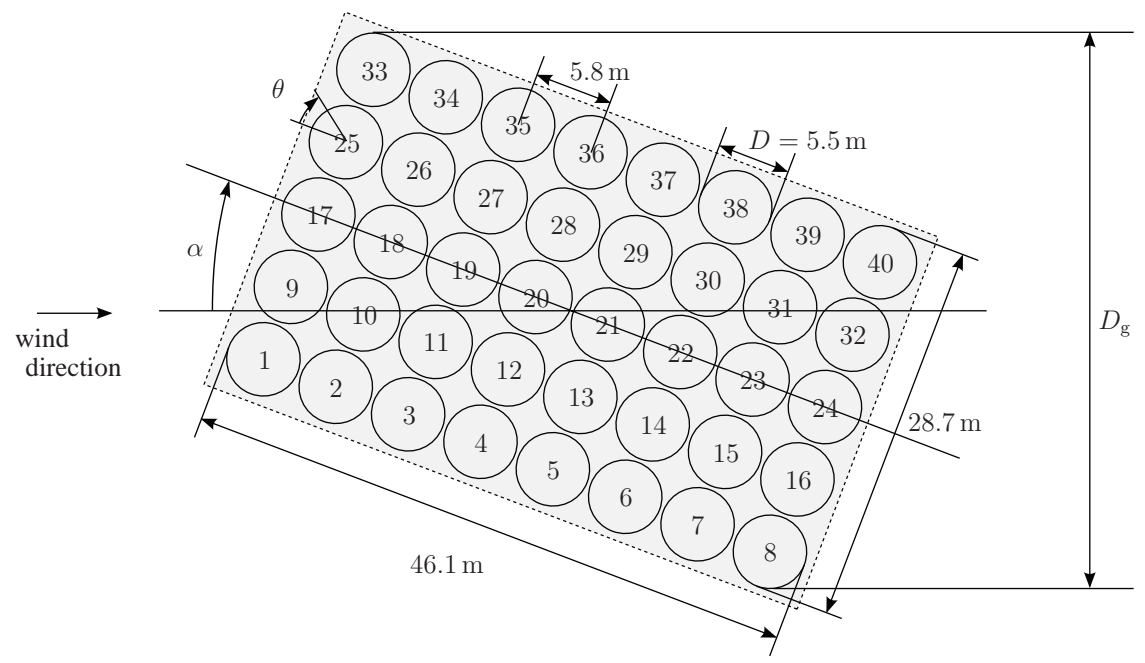

Figure 2. Plan view of the 8 by 5 silo group with numbering of the invididual silos. Dimensions are given as well as definitions for the angle of incidence $\alpha$ and the angle $\theta$ on the circumference of an individual cylinder. The contour of the rectangular building below the silo group is shown in grey. 


\section{STRUCTURAL OVALLING MODE SHAPES}

Ovalling deformation of a thin-walled shell structure is defined as a deformation of the cross section of the structure without bending deformation with respect to the longitudinal axis of symmetry [1]. The ovalling mode shapes for the thin-walled empty silos (diameter $D=5.5 \mathrm{~m}$ and wall thickness $\mathrm{t}=0.07 \mathrm{~m}-0.10 \mathrm{~m}$ varying along the height of the silo) are referred to by a couple $(m, n)$ where $m$ denotes the half wave number in the axial direction and $n$ is the number of circumferential waves (figure 3 ).

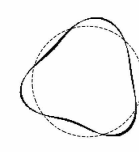

(a)

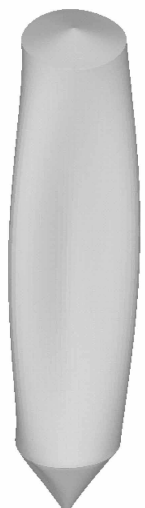

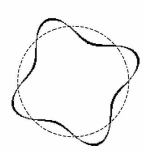

(b)

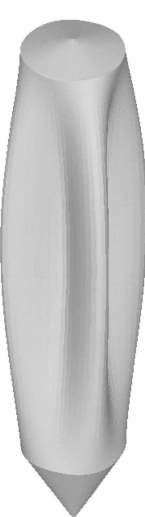

(c)
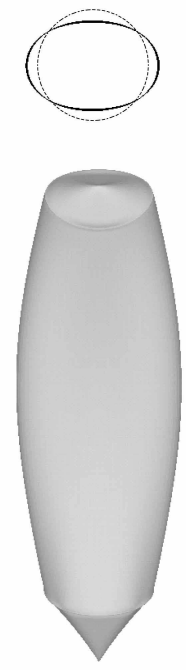

Figure 3. Selected ovalling eigenmodes of a single silo: (a) mode $\Phi_{1}$ at $3.93 \mathrm{~Hz}$, (b) mode $\boldsymbol{\Phi}_{3}$ also at $3.93 \mathrm{~Hz}$ and (c) mode $\Phi_{11}$ at $7.76 \mathrm{~Hz}$ [3].

The mode shapes are determined through FE analysis of the silo structure in the Abaqus software package [2]. To accomodate an easy transfer of the aerodynamic pressures on the silo walls to the mesh of the structural model (see section 4), the mesh of the FE model was chosen conforming to the mesh on the silo walls in the 3D CFD simulations (figures $4 \mathrm{a}$ and 9). Since the cone at the bottom of the silo structures is covered by a rectangular building below the silo (figure 9), a separate mesh is defined for this part of the structure, compatible with that of the superstructure. Aluminium shell elements with linear FE interpolation functions are used for all silo elements (density $\rho=2700 \mathrm{~kg} / \mathrm{m}^{3}$, Young's modulus $E=67.6 \mathrm{GPa}$ and Poisson ratio $\nu=0.35$ ). The mode shapes and natural frequencies of the silo structure are the solutions of the following generalized eigenvalue problem:

$$
\mathbf{K} \Phi=\omega^{2} \mathbf{M} \Phi
$$

where $\mathbf{M}$ and $\mathbf{K}$ are the mass and stiffness matrix respectively and $\omega=2 \pi f_{\text {eig }}$ with $f_{\text {eig }}$ the eigenfrequencies of the structure. The orthonormal base of eigenvectors (mode shapes) $\Phi$ of this eigenvalue problem are mass normalized. For validation, the computed natural frequencies and corresponding mode shapes are compared with results from a similar analysis of Dooms et al. [3] where another FE solver (Ansys Structural [4]) and a different mesh design (figure 4b) were used. Good agreement is found between both models for all mode shapes. Table 1 shows this agreement for the lowest natural frequencies. 


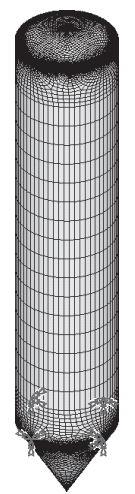

Figure 4. FE mesh design in (a) Abaqus and (a) Ansys Structural [3]

Table 1. Structural natural frequencies $f_{\text {eig }}$, computed in two different FE packages.

\begin{tabular}{cccc} 
& & Abaqus & Ansys Structural [3] \\
$\boldsymbol{\Phi}_{i}$ & $(m, n)$ & $f_{\text {eig }}[\mathrm{Hz}]$ & $f_{\text {eig }}[\mathrm{Hz}]$ \\
\hline $\boldsymbol{\Phi}_{1,2}$ & $(1,3)$ & 3.94 & 3.93 \\
$\boldsymbol{\Phi}_{3,4}$ & $(1,4)$ & 3.95 & 3.93 \\
$\boldsymbol{\Phi}_{5,6}$ & $(1,5)$ & 5.29 & 5.28 \\
$\boldsymbol{\Phi}_{7,8}$ & $(1,5)$ & 5.60 & 5.59 \\
$\boldsymbol{\Phi}_{9,10}$ & $(1,6)$ & 7.47 & 7.38 \\
$\boldsymbol{\Phi}_{11}$ & $(1,2)$ & 7.77 & 7.76
\end{tabular}

The visually detected pattern of vibrations at the lee side of the silo group during the 2002 storm seems to have excited ovalling mode shapes $(1,3)$ and $(1,4)$, corresponding to the lowest natural frequencies of the silo structure. Furthermore, measurements during normal wind loading have shown that eigenmodes with 3 or 4 circumferential wavelength have the highest contribution to the response of the silos [3].

\section{WIND FLOW AROUND THE SILO GROUP}

To investigate the highly turbulent wind flow around the silo group, both $2 \mathrm{D}$ and $3 \mathrm{D}$ CFD simulations have been performed. While the 2D simulations mainly aim to study the influence of the angle of incidence of the wind flow on the location where ovalling vibrations can be observed, the 3D simulations are performed to incorporate 3D flow effects into the analysis and to assess the validity of the conclusions of the 2D simulations. Since the emphasis in this paper is on the analysis of the aerodynamic pressures on the silo walls, the applied CFD techniques and verification and validation of the simulation results will only be briefly discussed. An important and interesting issue concerning the simulation of highly turbulent wind flow at the inlet of the domain is expanded upon in this section.

\subsection{Computational procedure}

The finite volume method is used for the discretization of the governing incompressible Navier-Stokes equations in the CFD simulations. Since the focus is on the structural 
response in this analysis, it is unnecessary to resolve all details of turbulent fluctuations in the flow. Thus, instead of resolving all turbulent scales in a direct numerical simulation (DNS), techniques have been developed for the numerical treatment of turbulence, e.g. the Reynolds averaged Navier-Stokes (RANS) procedure or large eddy simulations (LES). The choice of a particular technique depends on the complexity of the geometry, the turbulence levels and computation time. For the highly turbulent, external aerodynamic flow of the present case, DNS or LES simulations are computationally too demanding, especially since very accurate near-wall flows are required to get a good prediction of the aerodynamic pressures on the silo walls. It is therefore advisable to use the near-wall modeling of the RANS techniques.

For the 2D simulations, the unsteady RANS (or URANS) discretized set of equations is solved in the Ansys Fluent software package [5], using the hybrid shear-stress transport (SST) turbulence model. In 3D on the other hand, delayed detached eddy simulations (DDES) are performed in Ansys Fluent [6]. DES models are often refered to as hybrid LES/RANS models since the URANS modeling of the boundary layer flow in the near-wall region is combined with the LES approach in the separated regions, where large unsteady turbulence scales are dominant. In the delayed DES approach, a shielding function is used to ensure that RANS is applied in the entire boundary layer since a sole geometrical separation of RANS and LES regions based on mesh size has been shown to be insufficient. For the shielding function, the blending functions of the SST turbulence model are used [5]. For all simulations, a coupled pressure-based calculation with a second-order interpolation of the pressure, a second-order upwind interpolation of the turbulent kinetic energy $k$ and the specific dissipation rate $\omega$ and a second-order implicit, unconditionally stable, time stepping method are used. For the discretization of all transport equations, second-order upwind interpolation is used in the 2D URANS simulations while a bounded central differencing scheme is used in 3D DDES.

It is very important to be conscious of the specific properties of the modeling approaches used in the simulations. In 2D URANS simulations for instance, very coherent vortex structures are artificially preserved in the vortex street in the wake of a separated flow due to the time-averaging operation of turbulence and the absence of a third spatial dimension (figure 5). This results in slightly higher drag coefficients in 2D URANS with respect to 3D DDES simulations where vortex structures in the wake of a structure are solved more realistically and break up more quickly as they are carried downstream (figure 6). In order to draw correct conclusions from the simulation results, the potential inaccuracies of a certain modeling technique have to be taken into account during the interpretation of the simulations.

\subsection{Computational domain and boundary conditions}

The boundaries of the computational domain should be sufficiently far from the zone of interest in the centre of the domain (i.e. where the silo structures are modeled). Several guidelines are available in the literature with rules of thumb for the size of the computational domain and the boundary conditions in 2D and 3D. Specific difficulties with the inlet of the computational domain in 2D and 3D are mentioned in the next section (see 3.3).

Behr et al. [7] suggest that in 2D simulations of the flow around a single cylinder with diameter $D$, a distance of at least $8 D$ to the inlet of the domain and the lateral boundaries and a distance of $22.5 D$ to the outlet should be used. Therefore, in the present simulations slightly 


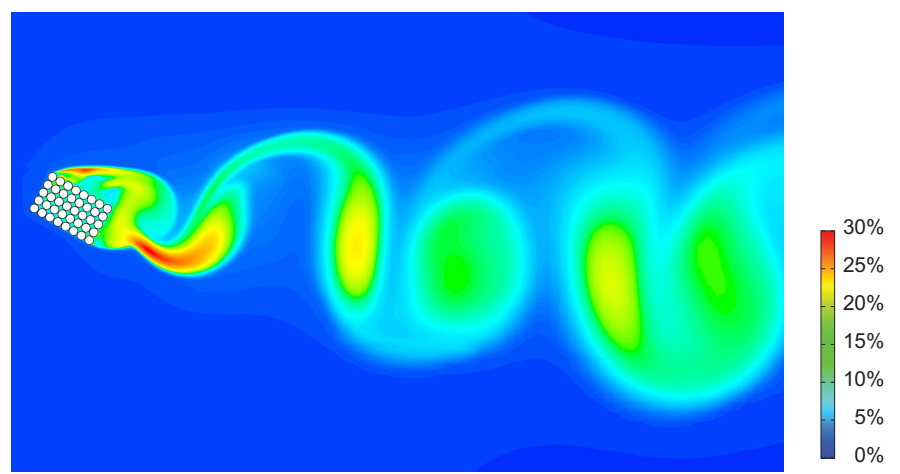

Figure 5 . Turbulence intensity in the flow around the 8 by 5 cylinder group, calculated with 2D URANS, for an angle of incidence $\alpha=30^{\circ}$ at $t=77.0 \mathrm{~s}$. The corresponding vortex shedding frequency is $f_{\mathrm{vs}}=0.25 \mathrm{~Hz}$.

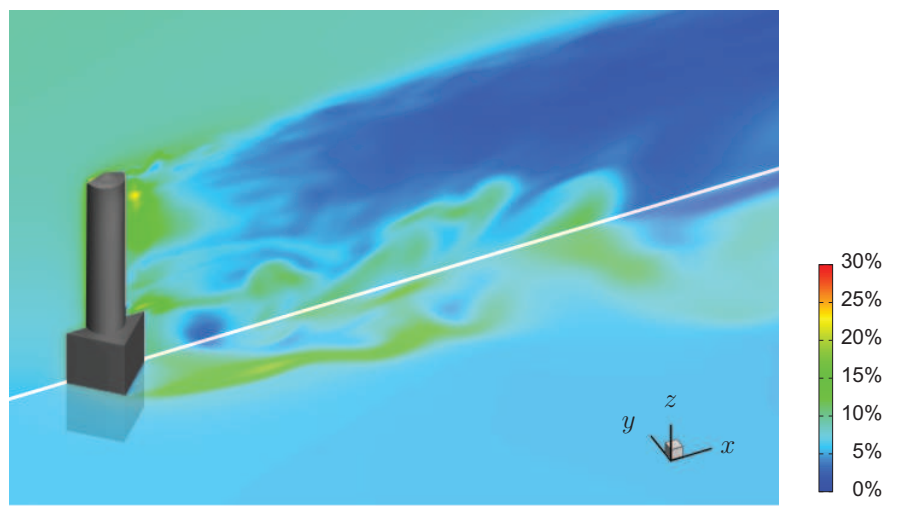

Figure 6. Turbulence intensity in the flow around a single silo structure, calculated with 3D DDES, for an angle of incidence $\alpha=45^{\circ}$ at $t=60.0 \mathrm{~s}$. The turbulence intensity is shown in a vertical plane ( $x z$-plane with $y=0 \mathrm{~m}$ ) and a horizontal plane (parallel to the $x y$ plane with $z=17 \mathrm{~m})$.

larger values of $9 D$ and $30 D$ are adopted for the case of a single cylinder in cross flow and, equivalently, $9 D_{g}$ and $30 D_{g}$ for the entire 8 by 5 silo group, where $D_{g}$ represents the projected width of the silo group (2). The outlet boundary of the domain is modeled as a pressure outlet where the static pressure is set equal to a reference value and symmetry is imposed on the lateral boundaries of the domain.

Based on wind tunnel experiments, guidelines have been set up by the Architectural Institute of Japan (AIJ) for the size of the computational domain in 3D wind simulations [8]. For a single building model, the lateral and the top boundaries should be set $5 H$ or more away from the building and the outflow boundary should be located at least $10 \mathrm{H}$ behind the building where $H$ is the height of the building. Furthermore, the blocking ratio (i.e. building cross section/domain section) should be below 3\%. As shown in figure 7, slightly larger values of $6 \mathrm{H}$ and $11 \mathrm{H}$ are again used, resulting in a blocking ratio of $1.7 \%$. Similar to the 2D simulations, the outflow boundary is modeled as a pressure outlet while symmetry is imposed on lateral and top boundaries. The bottom of the domain and the walls of the structure are considered smooth and no-slip boundary conditions are applied. 


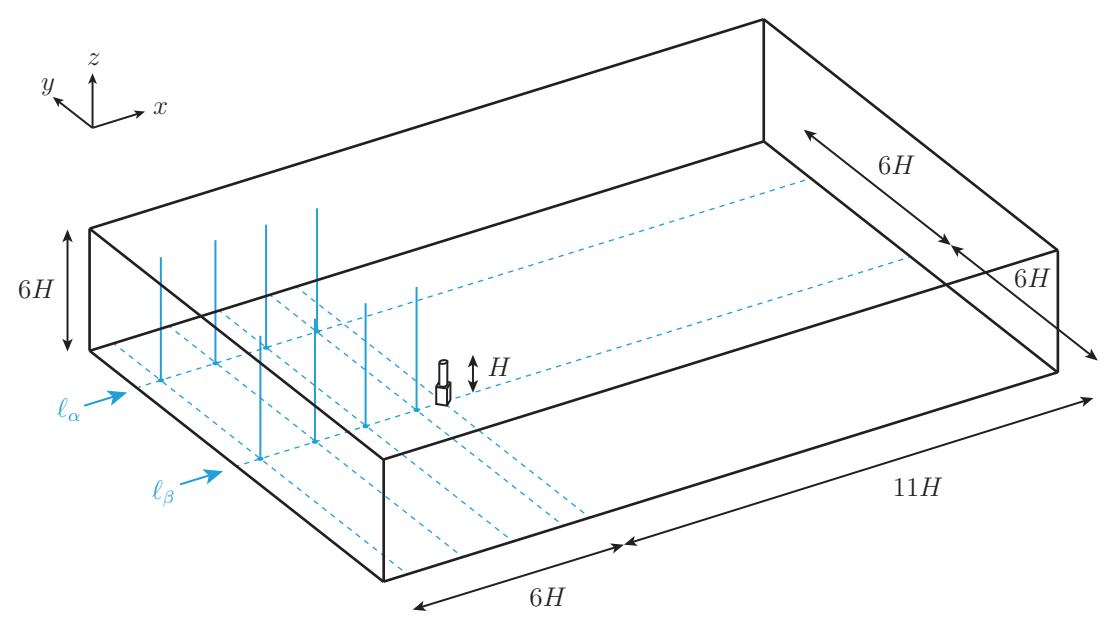

Figure 7. Dimensions of the 3D computational domain and global coordinate system, with origin at the bottom of the domain at the center of the structure.

\subsection{Inlet boundary condition}

Since the specific atmospheric conditions near the silo group were not monitored during the storm, approximative wind conditions have to be set up, based on the location of the group and mean wind velocities for storm conditions in design codes. Based on the Eurocode 1 design guidelines for wind loading [9], a mean wind velocity $v_{\infty}=31.8 \mathrm{~m} / \mathrm{s}$ is determined at half the height of the silos (approx. $z=30 \mathrm{~m}$ ), resulting in a post-critical wind flow at Reynolds number $\operatorname{Re}=v_{\infty} D / \nu=1.24 \times 10^{7}$. The global wind direction at the time of ovalling was at an angle of incidence of approximately $\alpha=30^{\circ}$.

In the 2D URANS simulations, the mean free stream velocity $v_{\infty}$ is imposed at the inlet along with turbulence intensity $\mathrm{Tu}=1 \%$ and turbulence length scale $l=0.06 D_{g}=1.8 \mathrm{~m}$. The latter is chosen as a percentage of a characteristic dimension of the problem (i.e. $D_{g}$ ) as proposed by Sak et al. [10]. In the atmospheric boundary layer (ABL), much larger turbulence quantities are usually found (e.g. up to $\mathrm{Tu}=20 \%$ on the earth surface [11]). However, while large turbulence length scales should be resolved in the simulations, they are not in URANS simulations where turbulence models are used instead. Imposing higher turbulence intensities, associated with large length scales results in excessive turbulence viscosity of the incident flow and yields unphysical results [12].

The typical logarithmic velocity and turbulence profiles of the ABL have to be imposed at the inlet of the 3D computational domain. As recommended by AIJ [8], a power law is used in the simulations as an alternative for the logarithmic profiles:

$$
\begin{aligned}
v_{x}(z) & =v_{R}\left(z / z_{R}\right)^{\alpha} \\
\operatorname{Tu}(z) & =0.1\left(z / z_{G}\right)^{-\alpha-0.05}
\end{aligned}
$$

where $v_{R}=v_{\infty}=31.8 \mathrm{~m} / \mathrm{s}$ is the reference velocity at a reference height $z_{R}=30 \mathrm{~m}$ (identical parameters as in the 2D simulations). The power coefficient $\alpha=0.14$ and gradient height of the $\mathrm{ABL} z_{G}=300 \mathrm{~m}$ are determined for terrain category 2 (open country) in the AIJ guidelines [8]. Based on these equations, ABL-profiles for velocity, turbulent kinetic energy $k$ and turbulence dissipation rate $\omega$ can be derived and imposed at the inlet. To simulate time 
dependent fluctuations, superimposed on these mean profiles, the vortex method is used as implemented in Fluent [6]. In the vortex method, time dependent inlet conditions are generated by adding a perturbation on a specified mean velocity profile via an imposed fluctuating vorticity field.

To obtain correct simulation results, it is important that the ABL profile is preserved from the inlet of the domain until it reaches the obstacle (i.e. the silo group). Figure 8 shows the mean horizontal wind velocity $v_{x}(z)$ at different locations in the computational domain (lines $\ell_{\alpha}$ and $\ell_{\beta}$ in figure 7). With full inlet turbulence, there is a significant deformation of the velocity profile approaching the building near the ground (grey curves in figure 8) due to a high momentum transfer by the fluctuating velocity field from the outer flow to the flow at ground level. Köse et al. [13] showed that by reducing the level of turbulent kinetic energy at the inlet (e.g. $0.5 k$ ), the inlet profile is preserved until it reaches the structure (black lines in figure 8). Indeed, on line $\ell_{\beta}$, the mean velocity profile is nicely preserved while on line $\ell_{\alpha}$ in the axis of the structure $(y=0 \mathrm{~m})$, the ABL-profile is deformed only in the last point $(x=-25 \mathrm{~m})$. This deformation is due to the vicinity of the silo structure $(H=41.66 \mathrm{~m})$ and is clearly different from the near-wall deformations observed when full turbulence is applied at the inlet.

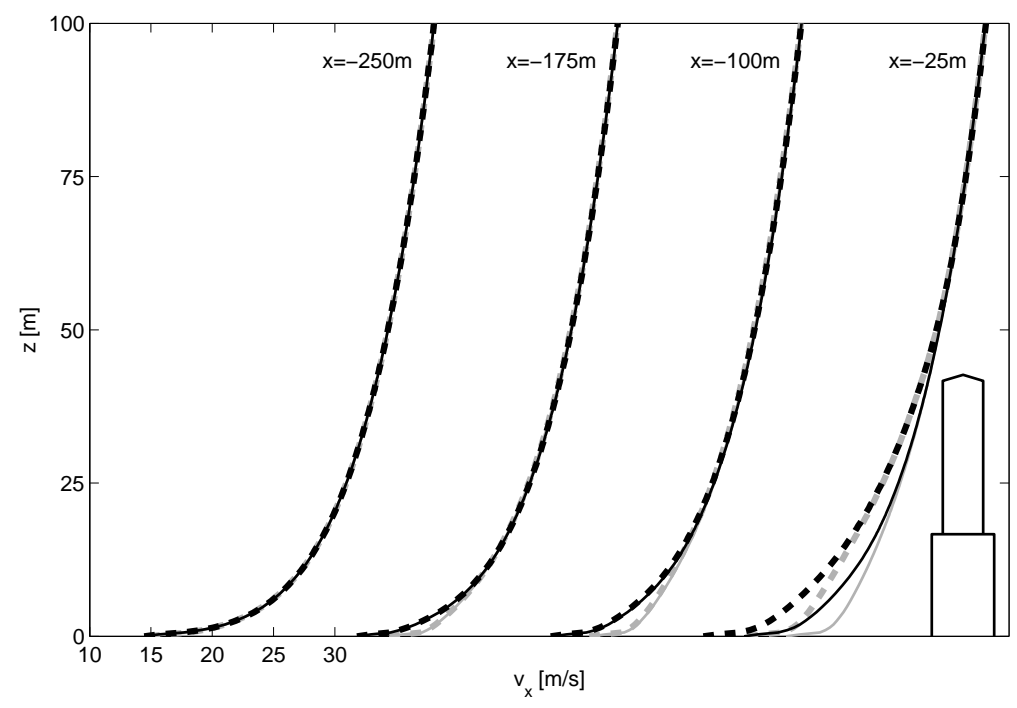

Figure 8. Deformation of the mean horizontal wind velocity profile $v_{x}(z)$ as it advances in the 3D DDES domain on a line in the axis of the structure (full lines, $y=0 \mathrm{~m}, \ell_{\alpha}$ in figure 7) and on a line next to the obstacle (dashed lines, $y=200 \mathrm{~m}, \ell_{\beta}$ in figure 7) for full inlet turbulence (1.0k, grey lines) and reduced inlet turbulence $(0.5 k$, black lines).

\subsection{Verification and validation of simulation results}

For these 2D and 3D transient calculations, both grid and time step independency have been checked. A time step of $\Delta t=0.005 \mathrm{~s}$ is applied in both 2D and 3D simulations. For the grid refinement, particular care has been taken to the near-wall treatment at the solid walls, since it is of primary importance that the pressures on the silo walls are accurately predicted. Full account of this extensive verification procedure for the $2 \mathrm{D}$ simulations is given in a previous publication of the authors [12]. A similar procedure was followed for the 3D 
simulations, with emphasis on the accurate modeling of the wall pressures. Figure 9 shows a detail of the mesh design for a 3D simulation of a silo structure where the rectangular building below the silo is oriented at an angle of incidence $\alpha=45^{\circ}$. This detail shows a clearly denser grid distribution on the silo walls compared to the grid density on the rectangular building below the structure where accurate wall pressures are less important.

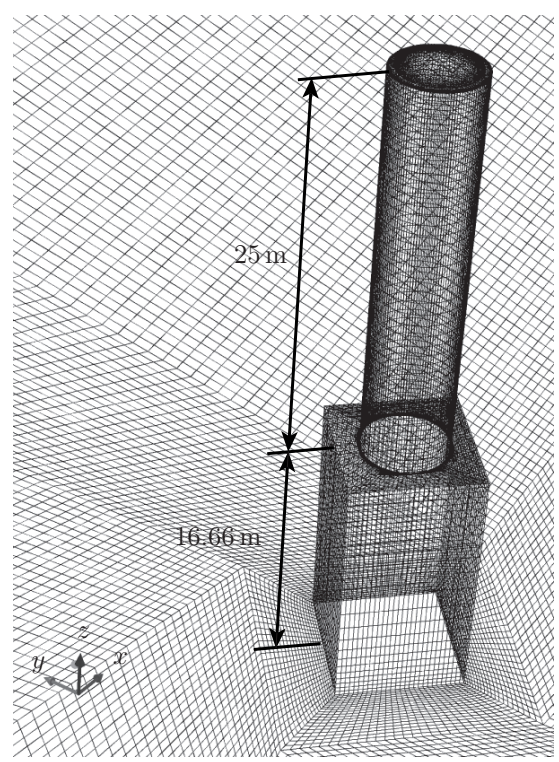

Figure 9. Detail of mesh design for 3D DDES of a single silo structure with the wind at an angle of incidence $\alpha=45^{\circ}$.

The validation of the highly turbulent wind flow around the silo group is a challenging task. No wind tunnel experiments or on-site measurements have been carried out for these particular silo structures. Therefore, the validation has been performed for the $2 \mathrm{D}$ simulation of a single cylinder at post-critical Reynolds numbers. Figure 10 shows good agreement between the present 2D simulation results for a single cylinder with experimental wind tunnel results gathered by Zdravkovich [14] and Shih et al. [15] for very high Reynolds numbers in the range of the present $\operatorname{Re}=1.24 \times 10^{7}$. The pressure coefficient $C_{\mathrm{p}}(\theta, t)$ along the circumference of a cylinder at a certain time $t$, presented in this figure, is defined as

$$
C_{\mathrm{p}}(\theta, t)=\frac{p(\theta, t)-p_{\infty}}{\rho v_{\infty}^{2} / 2},
$$

with $p_{\infty}$ the free stream pressure and $v_{\infty}$ the free stream velocity of the fluid. For the 2D simulations of the 8 by 5 cylinder group, results were qualitatively validated with the flow around bluff rectangular cylinders and the flow through tube bundles (e.g. in heat exchangers). Despite their geometrical resemblance, the simulations showed that the cylinder group can be treated neither as a tube array nor as a solid bluff body. A full account of this validation process of the 2D simulations is given in [12].

The validation for the 3D simulations is even more challenging. So far, validation could only be performed by comparing 3D simulation results with results from 2D simulations but a more rigorous validation is required. Therefore, wind tunnel experiments are currently being scheduled for the validation of the flow simulations around a single silo in ABL flow. 


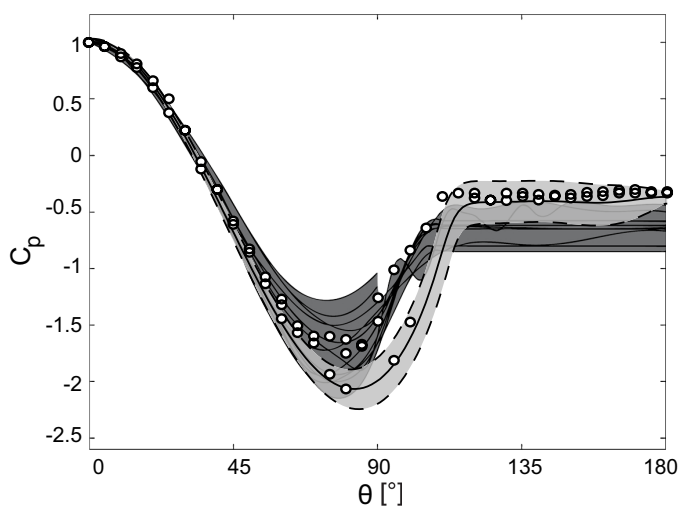

Figure 10. Comparison of maximal, minimal (dashed lines) and time averaged pressure coefficients $\bar{C}_{\mathrm{p}}(\theta)$ (solid line) for the flow around a single $2 \mathrm{D}$ cylinder at $\mathrm{Re}=1.24 \times 10^{7}$ with experimental results of Zdravkovich [13] for $0.73 \times 10^{7} \leq \mathrm{Re} \leq 3.65 \times 10^{7}$ (dark grey zone) and Shih et al. [14] at $\operatorname{Re}=0.8 \times 10^{7}(\mathrm{o})$.

Nevertheless, analysis of the dynamic 3D pressure distributions on the silo walls, presented in the next section, shows promising results.

\section{STRUCTURAL RESPONSE TO THE AERODYNAMIC PRESSURES}

\subsection{Application of aerodynamic pressures on FE silo model}

To investigate the onset of the wind induced ovalling vibrations, the study of the response of the silo structures to the aerodynamic pressures is very important. The aerodynamic pressure distribution $\mathrm{P}(\mathrm{t})$ due to the $3 \mathrm{D}$ wind flow around a single silo, with the rectangular building below at an angle of incidence $\alpha=45^{\circ}$, is determined and applied as an external transient load on the surface of the FE model of the silo structure described in section 2. As mentioned before, no interpolation of the pressure distribution output of the 3D fluid solver to the structural mesh is required since an identical mesh design is used for the silo walls in both fluid and structural solver.

A direct time integration scheme is used to solve the system of dynamic structural equations:

$$
\mathbf{M} \ddot{\mathbf{U}}(t)+\mathbf{C} \dot{\mathbf{U}}(t)+\mathbf{K U}(t)=\mathbf{P}(t)
$$

Rayleigh damping is assumed for the damping matrix $\mathbf{C}=\alpha_{R} \mathbf{M}+\beta_{R} \mathbf{K}$ with $\alpha_{\mathrm{R}}=0.495 \mathrm{~s}^{-1}$ and $\beta_{\mathrm{R}}=8.0 \times 10^{-4} \mathrm{~s}$, based on a constant damping ratio $\xi=0.02$ for the two lowest eigenmodes. This system of equations with a total of 74704 degrees of freedom can be significantly reduced by modal decomposition of the displacement vector $\mathbf{U}(\mathrm{t})=\mathbf{\Phi} \mathbf{X}(t)$ with $\mathbf{X}(\mathrm{t})$ the modal coordinates and subsequent projection onto the orthonormal base of eigenvectors $\Phi$ :

$$
\boldsymbol{\Phi}^{\mathrm{T}} \mathbf{M} \boldsymbol{\Phi} \ddot{\mathbf{X}}(t)+\boldsymbol{\Phi}^{\mathrm{T}} \mathbf{C} \boldsymbol{\Phi} \dot{\mathbf{X}}(t)+\boldsymbol{\Phi}^{\mathrm{T}} \mathbf{K} \boldsymbol{\Phi} \mathbf{X}(t)=\boldsymbol{\Phi}^{\mathrm{T}} \mathbf{P}(t)
$$

Depending on the number of eigenvectors (mode shapes) $\Phi$ included in the analysis (e.g. 50 in this analysis), the system of equations is reduced from 74704 degrees of freedom to only 50. As will be shown in the next paragraph, it is expected that only the eigenvectors with the 
lowest eigenfrequencies will contribute significantly to the dynamic response of the structures, which allows for a further reduction of the structural problem size once the natural frequencies and mode shapes have been determined.

Both approaches (direct time integration and modal superposition) yield qualitatively similar results, as shown in figure 11, with small differences in displacement in both approaches. An even more accurate coincidence between the two models can be obtained by using more than the current 50 eigenvectors in the orthonormal base $\Phi$ for the modal superposition. Energy methods are being used to determine the minimal number of eigenvectors that have to be taken into account.

It is important to mention that the calculated vibration amplitudes from this one-way coupling approach (max. approx. $95 \mathrm{~mm}$ ) are of the same order of magnitude as the observed vibration levels in the Antwerp silo group during the 2002 storm. Furthermore, the vibration pattern of the silo shell exciting mode shapes $(1,3)$ and $(1,4)$ in the present simulations corresponds well with the visually observed pattern of vibrations during the 2002 storm.

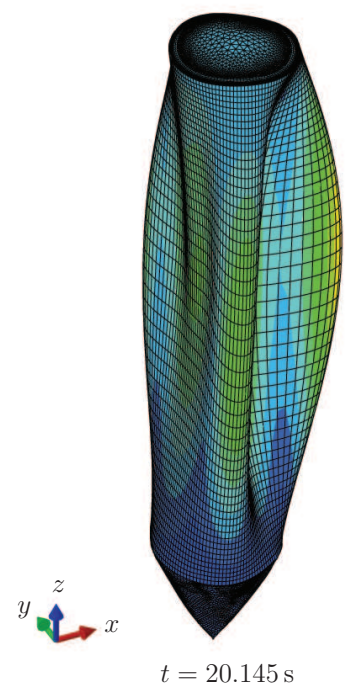

$t=20.145 \mathrm{~s}$

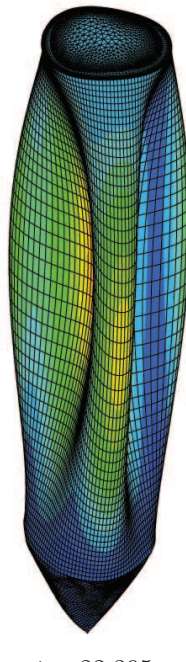

$t=22.395 \mathrm{~s}$ (b)

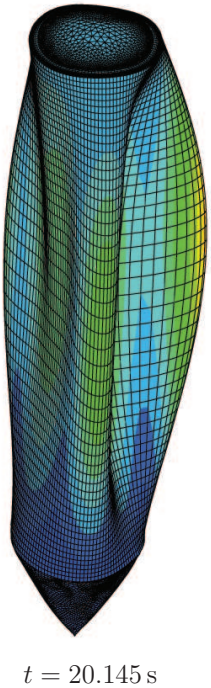

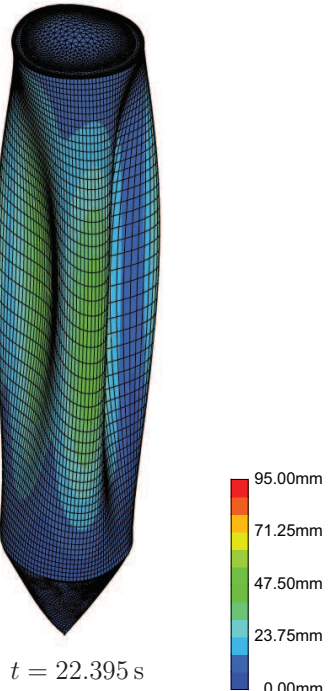

Figure 11. Deformation of the silo structure subjected to transient $3 \mathrm{D}$ wind pressures at $\mathrm{t}=$ $20.145 \mathrm{~s}$ and $\mathrm{t}=22.395 \mathrm{~s}$, solved by means of (a) direct time integration and (b) modal decomposition.

\subsection{Modal decomposition of aerodynamic pressures}

Based on the modal decomposition approach presented in the previous paragraph, it is interesting to examine the modal projection $\boldsymbol{\Phi}^{\mathrm{T}} \mathbf{P}(t)$ of the transient loads. The projection of the aerodynamic pressure distribution on the orthonormal base of mode shapes yields information on the modal contribution of the external loading to the dynamic structural response. The pressure distribution (figure 12) is more or less uniform along the height of the cylindrical shell and is quite similar to the pressure distribution found for the $2 \mathrm{D}$ cylinder case (figure 10): positive pressures are observed at the upwind side of the cylinder, gradually switching to negative pressures on the sides due to suction and evolving into a quite uniform base pressure at the lee side of the cylinder once the shear layer has detached from the cylinder surface. 
Modal decomposition of the total pressures $\mathbf{P}(t)$ results in large modal contributions to the dynamic response of mode shapes $(1,3),(1,4)$ and $(1,2)$, which seems logical considering the alternation of positive and negative pressures along the circumference of the silo.

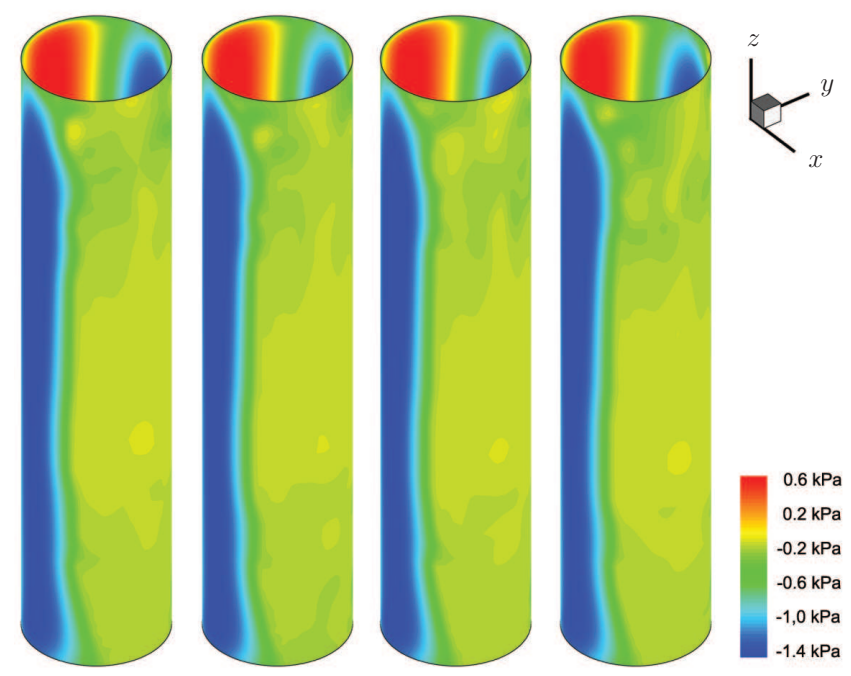

Figure 12. Pressure distribution $\mathbf{P}(t)$ on the silo walls for four time steps between $t=58.0 \mathrm{~s}$ and $t=59.5 \mathrm{~s}$.

However, vibrations can only be triggered by fluctuating pressures. Therefore, the total pressure $\mathbf{P}(t)$ on the silo walls has to be decomposed in time averaged pressures $\overline{\mathrm{P}}$, as a measure for the static deflection and fluctuating pressures $\mathbf{P}^{\prime}(t)$ as a measure for the dynamic excitation of the silos:

$$
\mathbf{P}^{\prime}(t)=\mathbf{P}(t)-\overline{\mathbf{P}}
$$

The fluctuating pressures $\mathbf{P}^{\prime}(t)$ are first modally decomposed and afterwards transformed to the frequency domain by means of a FFT algorithm, yielding $\boldsymbol{\Phi}^{\mathrm{T}} \mathbf{P}^{\prime}(f)$. The resulting frequency spectra for every mode shape are summarized in a mode shape-frequency spectrum, shown in figure 13. For better comparison with the analysis of the 2D simulation results, only the ovalling mode shapes with a half axial wavelength $(1, n)$ are shown in this figure. The colour of the band peaks in such spectra is a measure for the excitation level of the dynamic pressure loads on the silo surface, while the width is a measure for their steadiness. It can be observed that the frequency contribution rapidly decreases as the frequency increases, corresponding to measured wind spectra with typically low frequency components. Furthermore, the frequency content of the pressure fluctuations is quite uniform for all mode shapes and no specific dark narrow band peaks can be observed for a specific mode shape. This indicates a (more or less) uniform low frequency excitation of all mode shapes due to the fluctuating pressures. To confirm this conclusion, another method to distinguish the excitation of the different mode shapes, e.g. the energy transfer of the distributed pressure load to the different mode shapes, could be investigated. Furthermore, the amplitudes of the dynamic pressures $\mathbf{P}^{\prime}(t)$ are approximately one to two orders of magnitude smaller than the static pressures. They might hence give rise to visible vibrations, once they are superimposed on the static deformation of the silo structure. 


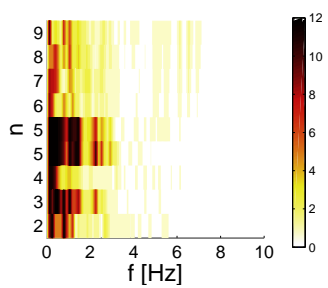

Figure 13. Mode shape-frequency spectrum of the modally projected fluctuating presures $\boldsymbol{\Phi}^{\mathrm{T}} \mathbf{P}^{\prime}(f)$ for mode shapes $(1, n)$ of a single 3D silo at angle of incidence $\alpha=45^{\circ}$.

For the 2D simulation results, a technique has been developped to project the pressure loads on the mode shapes of the silos [12]. First, the fluctuating pressure coefficient along the circumference of a cylinder at each time step is determined as follows:

$$
C_{\mathrm{p}}^{\prime}(\theta, t)=C_{\mathrm{p}}(\theta, t)-\bar{C}_{\mathrm{p}}(\theta)
$$

Subsequently, the fluctuating pressure coefficient $C_{\mathrm{p}}^{\prime}(\theta, t)$ is harmonically decomposed at every time step into a series of cosine functions with circumferential wavenumber $n$, corresponding to a geometrical approximation of the mode shapes at midheight of the axisymmetric structures (cfr. figure 3):

$$
C_{\mathrm{p}}^{\prime}(\theta, t)=\sum_{n=0}^{\infty} C_{\mathrm{p}}^{\prime n}(t) \cos \left(n \theta+\varphi_{n}\right) .
$$

After transformation to the frequency domain, wavenumber-frequency spectra of the amplitudes $C_{\mathrm{p}}^{\prime n}(f)$ can be created, similar to the mode shape-frequency spectra for the 3D results. In these wavenumber-frequency spectra, a dark narrow-band peak close to a structural natural frequency is very likely to excite resonance of the corresponding mode shape. The wavenumber-frequency spectrum for the $2 \mathrm{D}$ single cylinder simulation is shown in figure 14. Two dark narrow band frequency peaks can be observed at about $4 \mathrm{~Hz}$ and at its first harmonic (ca. $8 \mathrm{~Hz}$ ). Taking into account the circumferential wavenumbers $n$ on the vertical axis and the natural frequencies of the mode shapes, it is concluded the third and fourth circumferential eigenmodes $(1,3)$ and $(1,4)$ of the silos, both at a frequency of approximately $4 \mathrm{~Hz}$ would probably be excited by the dynamic wind pressures, as well as eigenmode $(1,2)$ at approximately $7.8 \mathrm{~Hz}$.

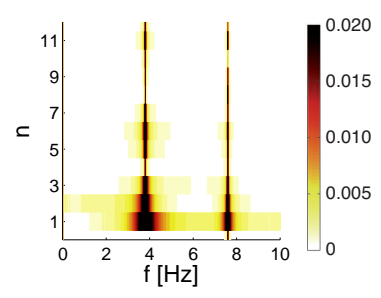

Figure 14. Wavenumber-frequency spectra of the amplitude $C_{\mathrm{p}}^{\prime n}(f)$ for the a single 2D cylinder.

For the 2D simulations of the entire silo group, wavenumber-frequency spectra can be determined for all 40 silos, as shown in figure 15 for $\alpha=30^{\circ}$. It is observed that the spectra at 
the upwind part of the group show no periodicities other than the low frequency contributions, related to the large vortex shedding in the wake of the group (cfr. $f_{\mathrm{vs}}$ in figure 5). Moving downwind, irregularities appear and in the downstream part of the group, two clear higher frequency peaks can be observed between $3 \mathrm{~Hz}$ and $4 \mathrm{~Hz}$ and also at approximately $6.5 \mathrm{~Hz}$. Similarly as for the single cylinder (figure 14), a forced resonant response corresponding to eigenmodes $(1,3)$ and $(1,4)$ is expected at the lee side corners of the silo group. This corresponds to the observed pattern of ovalling vibrations with three and four circumferential wavelengths. The same conclusions are found for other angles of incidence $\alpha$.

\section{CONCLUSIONS}

In order to clarify the observed ovalling vibrations in a group of 8 by 5 silos during a storm, 2D and 3D CFD simulations have been performed to numerically predict the aerodynamic pressure distributions on the silo walls. An important issue in CFD simulations is the application of correct turbulence parameters at the inlet of the computational domain. In both 2D and 3D simulations, reduced turbulence levels have to be imposed at the inlet of the domain to prevent unphysical results. The 3D pressure distribution on the silo walls has been applied as a transient external load on a 3D finite element model of a silo. Modal decomposition techniques are used to reduce the size of the structural problem. Vibration amplitudes resulting from this one-way coupling approach are in the same order of magnitude as the observed vibration levels in the Antwerp silo group during the 2002 storm. Modal projection of the $3 \mathrm{D}$ load field is performed to determine the excitation of each ovalling mode shape in the dynamic structural response. For the 2D simulations, a similar technique of harmonic decomposition is used to approximate the ovalling mode shapes in a $2 \mathrm{D}$ plane. The results in 2D indicate a forced, resonant structural response which corresponds to the observed pattern of ovalling vibrations while in 3D it is currently only observed that the ovalling mode shapes might be excited by the transient pressures, without particular preference for one or more ovalling modes. Energy methods are being used in further research to determine the ovalling modes that might be preferentially excited.

\section{Acknowledgements}

The research in this paper has been performed within the frame of the FWO project G.0275.08 "Efficient analysis of fluid-structure interaction problems in structural dynamics". The second author is a post-doc fellow of the Research Foundation Flanders (FWO). The support of FWO is gratefully acknowledged.

\section{References}

[1] M.P. Païdoussis, S.J. Price, and H.C. Suen. Ovalling oscillations of cantilevered and clamped-clamped cylindrical-shells in cross flow: An experimental-study. Journal of Sound and Vibration, 83(4):533-553, 1982.

[2] Abaqus 6.10. User's Manual. Dassault Systèmes Simulia Corp., 2010. 


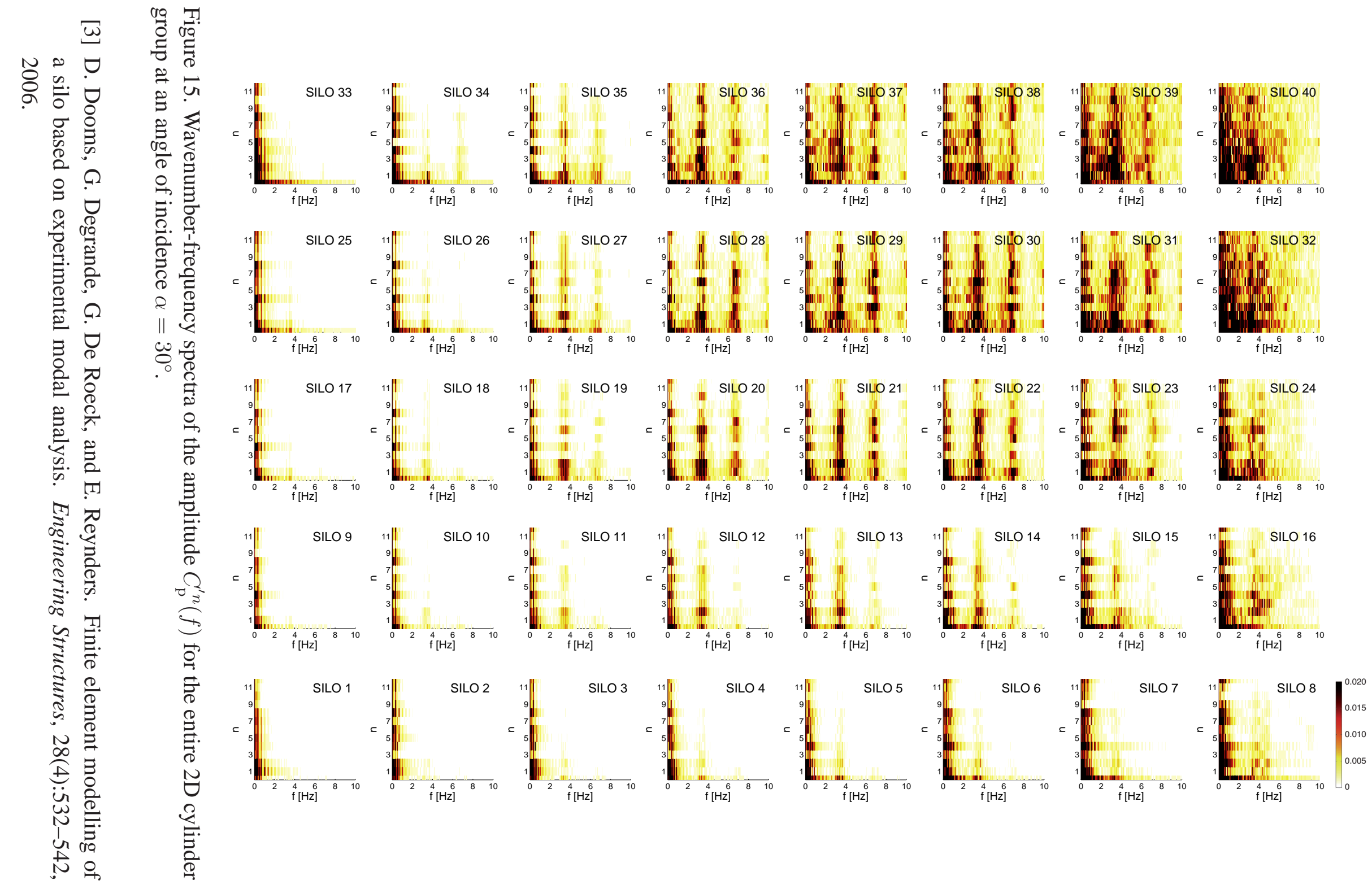


[4] Ansys 8.1. User's Manual. Ansys Inc., March 2004.

[5] FLUENT 12.0. User's Guide. Ansys Inc., April 2009.

[6] FLUENT 13.0. User's Guide. Ansys Inc., 2010.

[7] M. Behr, D. Hastreiter, S. Mittal, and T.E. Tezduyar. Incompressible flow past a circular cylinder: dependence of the computed flow field on the location of the lateral boundaries. Computer Methods in Applied Mechanics and Engineering, 123(1-4):309-316, 1995.

[8] Y. Tominaga, A. Mochida, R. Yoshie, H. Kataoka, T. Nozu, M Yoshikawa, and T. Shirisawa. AIJ guidelines for practical applications of CFD to pedestrian wind environment around buildings. Journal of Wind Engineering and Industrial Aerodynamics, 96:17491761, 2008.

[9] BIN. NBN EN 1991-1-4:2005 Eurocode 1: Actions on structures - Part 1-4: General actions - Wind actions. Belgisch Instituut voor Normalisatie, April 2005.

[10] C. Sak, R. Liu, D.S.-K. Ting, and R.W. Rankin. The role of turbulence length scale and turbulence intensity on forced convection from a heated horizontal circular cylinder. Experimental Thermal and Fluid Science, 31(4):279-289, 2007.

[11] ESDU. Data Item No. 85020: Characteristics of atmospheric turbulence near the ground. Part II: single point data for strong winds (neutral atmosphere). Engineering Science Data Unit, London, UK, 1985.

[12] J. Hillewaere, D. Dooms, B. Van Quekelberghe, J. Degroote, J. Vierendeels, G. De Roeck, G. Lombaert, and G. Degrande. Unsteady Reynolds averaged NavierStokes simulation of the post-critical flow around a closely spaced group of silos. Journal of Fluids and Structures, 30:51-72, 2012.

[13] D. Köse, D. Fauconnier, and E. Dick. ILES of flow over low-rise buildings: Influence of inflow conditions on the quality of the mean pressure distribution prediction. Journal of Wind Engineering and Industrial Aerodynamics, 99:1056-1068, 2011.

[14] M.M. Zdravkovich. Flow Around Circular Cylinders, Volume 1: Fundamentals. Oxford University Press, Oxford, England, 1997.

[15] W.C.L. Shih, C. Wang, D. Coles, and A. Roshko. Experiments on flow past rough circular cylinders at large Reynolds numbers. Journal of Wind Engineering and Industrial Aerodynamics, 49(1-3):351-368, 1993. 